\section{LA NOSTALGIA EN LA PRODUCCIÓN URBANA: LA DEFENSA DE BARRIOS EN SANTIAGO DE CHILE'}

Clément Colin²

\section{Resumen}

En los últimos años, la ciudad neoliberal se ha transformado en un tema central en los estudios urbanos. Uno de los aspectos analizado es la extensión de la presión inmobiliaria acompañada por la renovación urbana que consiste en destruir y reconstruir de manera permanente la ciudad. Sin embargo, son escasos los estudios que se interesan en la manera en que esos procesos son vividos y percibidos en la cotidianidad por sus habitantes, para desde ahí establecer un punto de vista crítico. Partiendo de esta constatación, el artículo propone interrogar la nostalgia gatillada por los cambios urbanos a partir del estudio de la movilización

\section{NOSTALGIA IN URBAN PRODUCTION: THE DEFENSE OF NEIGHBORHOODS IN SANTIAGO, CHILE ${ }^{1}$}

Clément Colin ${ }^{2}$

\section{Abstract}

The neoliberal city has recently become a central subject of study in urban studies. One of the aspects analyzed is the extension of the pressure exerted by the real estate market and urban renewal, an activity based in the constant destruction and reconstruction of cities. However, there are few studies focused on how these processes are experienced and perceived by dwellers within the context of their daily lives. Through the study of social conflicts against specific urban projects in defense of neighborhoods in Santiago Centro, Chile, this paper explores the nostalgia due to 
de habitantes contra los proyectos inmobiliarios y por la defensa de su barrio en Santiago Centro (Chile). El artículo se basa en un trabajo de campo de corte etnográfico realizado en 2014 y 2015 con grupos de habitantes involucrados en la defensa y la revalorización patrimonial del barrio Matta Sur. Con este trabajo se busca analizar y cuestionar la presencia y la materialización de la nostalgia en el espacio y sus usos y funciones socio-culturales actuales en la producción de la ciudad.

PALABRAS CLAVE: NOSTALGIA, PRODUCCIÓN URBANA, PATRIMONIO, BARRIO.

Recibido: 24-02-2017.

Aceptado: 31-08-2017.

1 El autor agradece el apoyo financiero de la Pontificia Universidad Católica de Valparaíso a través del proyecto ID Iniciación 039.446/2017, a partir de lo cual se formuló el marco teórico y el apoyo financiero del CEDEUS CONICYT/FONDAP 15110020, dentro del cual se realizó el trabajo de campo entre 2014 y 2015.

2 Chile. Profesor asociado de la Escuela de Trabajo Social, Pontificia Universidad Católica de Valparaíso. Correo electrónico: clement.colin@pucv.cl urban changes in the past. This research is based on an ethnographic field work conducted from 2014 to 2015 with groups of dwellers involved in the defense and promotion of the heritage value of the Matta Sur neighborhood. The aim of this paper is to analyze and question the use, presence and emergence of nostalgia within the space dimension and its current socio-cultural role in the production of cities.

KEYWORDS: NOSTALGIA, URBAN PRODUCTION, HERITAGE, NEIGHBORHOOD.

Received: 24-02-2017.

Accepted: 31-08-2017.

1 The author acknowledges the financial support provided by the Pontifical Catholic University of Valparaíso through Project $039.446 / 2017$, which enabled the development of the theoretical framework of this research. Appreciation is also extended to the financial support provided by CEDEUS CONICYT/FONDAP 15110020, which enabled the development of the fieldwork phase of this study during the period 2014 to 2015.

2 Chile. Associate Professor, School of Social Work, Pontifical Catholic University of Valparaíso. Email: clement.colin@pucv.cl. 


\section{Introducción}

En América Latina, en los últimos años, gran parte de la literatura sobre lo urbano se ha interesado en las formas socio-espaciales de la ciudad neoliberal. Concepto utilizado para interrogar la ciudad contemporánea, hace referencia a las políticas urbanas que se piensan y se aplican a escalas múltiples desde lo local hasta lo global (Hidalgo y Janoschka, 2014) y que se basan, por un lado, en la desregulación de los mercados y el retiro del Estado y, por otro lado, en la financiación de la economía, la especulación y la plusvalía urbana (Rodríguez y Rodríguez, 2009). En Chile, uno de los aspectos que ha sido estudiado es la extensión y el fortalecimiento de la presión inmobiliaria, acompañada por la liberalización del mercado del suelo (Mattos, 2002 y 2010; López, Gasic y Meza, 2012). Eso se traduce generalmente en la renovación urbana, que consiste en destruir y reconstruir de manera permanente la ciudad y que conduce muchas veces a una densificación del tejido urbano y a una verticalización de la ciudad, como por ejemplo en Santiago Centro desde los años 1990 (Hidalgo, 2010; Contreras, 2011). Para analizar los procesos de la renovación urbana como dimensión constituyente de la ciudad neoliberal (Harvey, 1990), los autores privilegian enfoques institucionales y/o estructuralistas. No obstante, escasos son los análisis que se interesan en la forma en la cual estas evoluciones son vividas y percibidas por sus habitantes.
Además, los cambios no son solamente materiales, sino que también involucran los estilos de vida y las costumbres. Por lo tanto, siguiendo la línea de pensamiento de Lefebvre (1974), según la cual la producción del espacio se fundaría también en sus dimensiones vividas y percibidas, el artículo enfatiza en la manera en que los habitantes viven, piensan y se piensan en el cambio socio-espacial permanente de la ciudad neoliberal.

Para abordar esta problemática, se interroga las dimensiones socio-espaciales de la nostalgia gatillada por los cambios urbanos, a partir del estudio de la movilización de habitantes contra los proyectos inmobiliarios y por la defensa de su barrio en Santiago Centro (Chile). Iniciado en los años 2000, este fenómeno se está difundiendo y ampliando en todas las comunas de Santiago y en Chile. Los grupos movilizados tienen un doble objetivo. El primero es defender el barrio de su posible destrucción debido a la multiplicación de proyectos inmobiliarios que ocurre en esta parte de la ciudad desde los años noventa. El segundo es revalorizar sus estilos de vida de barrio que consideran como patrimoniales. Comprender y analizar estos procesos a través de la idea de nostalgia permite abordar un problema de investigación mucho más amplio que el conflicto local aquí descrito: se quiere cuestionar la dimensión sensible y la experiencia afectiva del cambio urbano. Frente a los discursos desarrollistas de los poderes públicos y de los promotores inmobiliarios, los discursos nostálgicos y 
las prácticas colectivas de los habitantes se refieren a "futuros pasados", es decir "momentos de proyección y anticipación que subsisten en la forma de recuerdos, cuya evocación reactiva esperas a la vez antiguas y presentes" (Lachenal y Mbodj-Pouye, 2014, p. 6-7)3. A partir de un trabajo de campo de corte etnográfico realizado en 2014 y 2015 en un barrio de Santiago Centro -Matta Sur- con grupos de habitantes involucrados en su defensa y su revalorización patrimonial, se busca interrogar la presencia y la materialización de la nostalgia en el espacio y sus usos y funciones socio-culturales actuales. Se interroga así la nostalgia no solamente como sentimiento sino también como práctica y discurso utilizado en el presente para pensar otro futuro.

El artículo defiende la idea de que la nostalgia, en tanto construcción social, cultural y política, participa en la producción del espacio urbano. Se quiere mostrar, de esta forma, que la nostalgia puede ser a la vez un sentimiento, un discurso y una práctica de lucha contra la ciudad neoliberal actual, que conduce a las personas que la sienten a utilizarla como herramienta de apropiación del espacio. La demostración se organiza en cinco partes. En la primera se aborda la nostalgia desde la teoría como construcción socio-cultural anclada en el espacio. En la segunda parte se explica cómo la nostalgia ha sido identificada durante el trabajo de campo y cómo se ha elaborado como categoría de análisis. La tercera parte cuestiona las condiciones socioespaciales de emergencia del sentimiento de nostalgia en los habitantes movilizados en el contexto de la neoliberalización de la ciudad. En la cuarta parte se interroga la manera en que la nostalgia se construye entre participantes como discursos y prácticas comunes de los grupos estudiados. Y la última parte se focaliza en la nostalgia como herramienta de apropiación material e ideal del espacio barrial por los habitantes movilizados.

\section{La nostalgia como sentimiento, práctica y discurso en y sobre la ciudad}

En los últimos años del siglo XX se ha difundido progresivamente un gusto nostálgico por los objetos y las prácticas del pasado en campos tan diversos como el nacionalismo, las políticas patrimoniales, el consumismo, el turismo de masas, la cultura popular o los movimientos sociales. Palabra creada en el siglo XVII por el suizo Johannes Hofer para caracterizar un fenómeno médico, la nostalgia fue durante mucho tiempo considerada una patología psicológica (Starobinski, 1966). Nostalgia tiene su origen en dos palabras griegas: nostos, el regreso,

Traducción del autor. 
y algos, el sufrimiento. Los síntomas incluían, entre otros, desánimo, melancolía e inestabilidad del estado emocional que, a veces, pueden conducir a anorexia, depresión y suicidio (Davis, 1979). Sin desarrollar toda la historia de la noción de nostalgia, se puede señalar que durante el siglo XIX perdió sus connotaciones médicas para adquirir significaciones sociales y valores asociados con el sentimiento de lamento de un espacio o de un tiempo desaparecido o cambiado (Angé y Berliner, 2015). Progresivamente, la nostalgia ha acaparado la atención en relevantes debates contemporáneos, siendo presentada como uno de los componentes de las sociedades inquietas (Tuan, 1977), vinculada con el aumento del miedo y de las incertidumbres (Appadurai, 1996) y con la percepción de inseguridad (Sennett, 1998) en un mundo que cambia cada vez más rápidamente (Bauman, 2007).

En la literatura, la nostalgia ha sido ocasionalmente criticada por su supuesta capacidad para falsificar o cambiar las narraciones históricas (Huyssen, 2003; Todorov, 2004) e idealizar un pasado (Lowenthal, 1998). Sin embargo, en este artículo se afirma que la nostalgia no tiene solamente que ver con una relación activa con un pasado para compensar una pérdida (Pickering y Keightley, 2006), ya que su experimentación y expresión permiten también "una crítica moral del presente y propone alternativas para afrontar los cambios sociales"
(Angé y Berliner, 2015, p. 5)4. En esta perspectiva, los registros nostálgicos y anti-nostálgicos además coexisten y a veces se confrontan en los mismos espacios. No existe una nostalgia sino nostalgias, a veces en conflicto (Berliner, 2012) pues no se vinculan con los mismos recuerdos y significaciones según los individuos. Por lo tanto, se busca aquí superar una concepción conservadora de la nostalgia para pensarla como fenómeno socio-cultural anclado en el presente. Se aborda la nostalgia como discurso y práctica socio-cultural (Stewart, 1988), partiendo de la hipótesis de que su experimentación respecto de un espacio social o físico se traduce en -y revela- prácticas y conductas sociales en la ciudad. Es por eso que no solamente se puede interrogar las funciones y los sentidos sociales de la nostalgia, sino también sus inscripciones espaciales.

La nostalgia toma distintas formas en el espacio urbano, siendo la reminiscencia y la rememoración las dos principales (Gervais-Lambony, 2012). La primera se vincula con una memoria involuntaria que se produce en las percepciones generadas desde el cuerpo y la mente, a partir de analogías hechas entre lo que se percibe en el momento actual y momentos pasados. Muchas veces, la nostalgia es asociada con la idea de viaje o de migración. Pero, se afirma aquí que se experimenta también sin desplazamiento. Los

4 Traducción del autor. 
ciudadanos hoy en día están afrontando una forma particular de nostalgia generada por los cambios en su entorno: es la "nostalgia moderna" ya descrita por autores como Baudelaire o Benjamin (Gervais-Lambony, 2012). Está directamente vinculada con la renovación urbana y la destrucción-reconstrucción que caracteriza a la mayoría de las ciudades en el mundo. En este contexto, algunos espacios u objetos son preservados porque son protegidos o bien porque no tienen interés para la plusvalía inmobiliaria. Son los revenants (fantasmas) en el sentido de Certeau (1980): objetos olvidados que llaman a la actualidad recuerdos del pasado y que provocan sentimientos y emociones en los sujetos afectados. Pueden ser sencillos, de pequeño tamaño, o pueden ser también lugares, actividades o prácticas. Son objetos en riesgo porque no están en adecuación con la ciudad neoliberal que los considera obsoletos, pero hacen muchas veces sentido para los habitantes. Destinados a desaparecer o ser fijados en el tiempo y espacio por medio de su protección, escasos son los que logran perpetuarse vivos y en actividad. En este sentido, su (re)activación revela muchas veces prácticas y discursos de resistencia contra las evoluciones presentes y sirven como recurso para defender ideas o concepciones de lo que debería ser la ciudad.
A diferencia de la reminiscencia, la rememoración es el resultado de un esfuerzo de memoria voluntario que induce un proceso de reconstrucción (Ricoeur, 2000; Todorov, 2004). No se trata entonces de una perpetuación o de una continuidad exacta de las prácticas "de antes", sino más bien de una reinvención o reinterpretación permanente a partir de recuerdos y memorias desde imaginarios de "como era antes". Esta nostalgia se puede caracterizar como "reflexiva" (Boym, 2001), porque tiene que ver con una reinterpretación o una reinvención de un pasado en el tiempo presente. Además aporta sustento, por ejemplo, a las prácticas patrimoniales en políticas públicas, donde la protección tiene que ver con una re-imaginación de un pasado relacionada con valores actuales y a veces con la mercantilización de las sensaciones nostálgicas. Por otra parte, la rememoración juega un rol de apoyo a prácticas sociales de resistencia cultural, donde la nostalgia se puede entender como un modo de afiliación social y de construcciones identitarias (Bissell, 2005; Berdahl, 2010; Berliner, 2012).

Los grupos estudiados dan cuenta de estos dos tipos de relación afectiva con el espacio y el tiempo. En este marco se busca mostrar cómo juegan un rol en la movilización de vecinos contra los proyectos inmobiliarios y cómo se usan como soportes para actuar colectivamente en el espacio barrial. 


\section{La nostalgia como categoría analítica}

La nostalgia ha sido estudiada de distintas formas. Mientras algunos autores la abordan a través de discursos y políticas (Angé y Berliner, 2015), otros lo hacen mediante la observación participante (Lachenal y Mbodj-Pouye, 2014). En esta línea, la geografía cultural ha abierto pistas de reflexión en el tema de las emociones a través de investigaciones y teorías sobre los valores del espacio y las experiencias sensoriales vinculadas con los lugares (Tuan, 1977; Rodaway, 1994). En este marco, desde los años 2000, algunos autores anglosajones anhelan la elaboración de "geografías emocionales" (Davidson, Bondi y Smith, 2007; Smith, Davidson, Cameron y Bondi, 2009; Hemer y Bundon, 2016) que sean capaces de dar cuenta de la dimensión emocional de nuestras relaciones con los lugares y, más ampliamente, de nuestra relación con el mundo y de la manera en que las emociones son producidas por las interacciones de las sociedades con su entorno. El artículo propone seguir esta línea de pensamiento metodológico sobre la manera de integrar y abordar las emociones y los sentidos como objetos científicos.

En el caso estudiado, la nostalgia surgió en el marco del trabajo de campo y se ha impuesto progresivamente como tema central para entender la movilización y las reivindicaciones de los grupos de habitantes. Distintos elementos empíricos han conducido a esta elección. Los discursos compartidos entre participantes en los grupos estaban centrados sobre lo que "era mejor antes" o "como era antes". El deseo de hacer comunidad estaba también casi omnipresente en las palabras y las alocuciones de los líderes y el lamento de los habitantes por haber perdido una sociabilidad que antes era el centro de su conversación. Por otro lado, la nostalgia no se ha revelado solamente en los discursos sino también en los comportamientos y las reacciones durante las reuniones y los encuentros. Se han podido destacar intensidades de reacción afectiva más fuertes a la evocación de momentos pasados. Además, los participantes querían reproducir prácticas culturales percibidas como tradicionales, tales como los rituales de encuentros y de saludos entre vecinos en el fin del día, la planificación de comidas juntos entre habitantes o el mantenimiento de eventos culturales tradicionales en el espacio público.

El artículo se funda en un trabajo etnográfico realizado entre 2014 y 2015 con dos grupos de habitantes del barrio Matta Sur, en el sur-este de la comuna de Santiago Centro. En el siglo XIX, este sector se componía de chacras en torno de caminos y calles fangosas. En el curso de ese mismo siglo se fue consolidando progresivamente con la llegada de poblaciones rurales. Este espacio era conocido por sus muy difíciles condiciones de vida y su gran pobreza (Ramón, 2000). Es durante el 
siglo XX que esta zona al sur de Santiago (hoy en el corazón del área metropolitana: ver figura 1) se transforma en un sector urbano por medio de proyectos de construcción privados. En el Censo de 2002, el barrio se componía de 51.217 habitantes, equivalente al $25.5 \%$ de la población de la comuna de Santiago (Ilustre Municipalidad de Santiago, 2013). Barrio obrero en la segunda parte del siglo XX, es hoy dominado por familias de estrato socio-económico medio y medio bajo. Matta Sur es un barrio predominantemente residencial (53.7\% de las propiedades tienen este uso), gran parte de los edificios son de un piso (70.9\%) y fueron construidos antes de 1950. Solamente el 2.8\% de los edificios fue construido entre 2001 y 2007 (Ilustre Municipalidad de Santiago, 2013). Hoy, el barrio interesa a los promotores por su ubicación. Es por eso que desde 2007, grupos de habitantes se movilizan para evitar el mismo destino que otros barrios de Santiago.

Los dos grupos estudiados son el "Comité de Defensa del Barrio Matta Sur", creado en 2007, y el "Centro Cultural Patrimonio Matta Sur", fundado en 2009. Estas agrupaciones de defensa barrial no son las únicas del barrio, pero son las más organizadas y activas. Cada una posee entre treinta y cuarenta habitantes del barrio $^{5}$, que pertenecen en gran mayoría a las antiguas familias del barrio.
Los participantes pueden ser considerados como los establecidos, en el sentido de Elias y Scotson (1966). Son personas que han vivido toda su vida o gran parte de ella en el barrio y a veces en la misma casa. Se consideran como los herederos de los valores y estilos de vida de sus padres y abuelos. Las mujeres son dueñas de casa de aproximadamente cincuenta años, generalmente casadas con hombres de profesión intermedia (obreros o empleados). Otra parte importante de los participantes son jubilados (hombres y mujeres). Es también posible encontrar personas menores de cuarenta años interesadas por los proyectos del barrio y que viven con su familia o cerca de su familia en el barrio. Son grupos conducidos por personas de estrato socio-económico medio (arquitectos, profesores o trabajadores en el medio cultural), que saben movilizar sus recursos sociales y son capaces de adaptar sus discursos tanto para los habitantes como para las autoridades públicas. Estos líderes son conocidos por la municipalidad y las instituciones estatales, e incluso muchas veces son solicitados por los periodistas para abordar temas vinculados con el patrimonio y la defensa cultural de los barrios.

$5 \quad$ No existe registro oficial y muchos de los participantes están presentes en los dos grupos. 
FIGURA 1. UBICACIÓN DEL BARRIO MATTA SUR EN SANTIAGO DE CHILE.

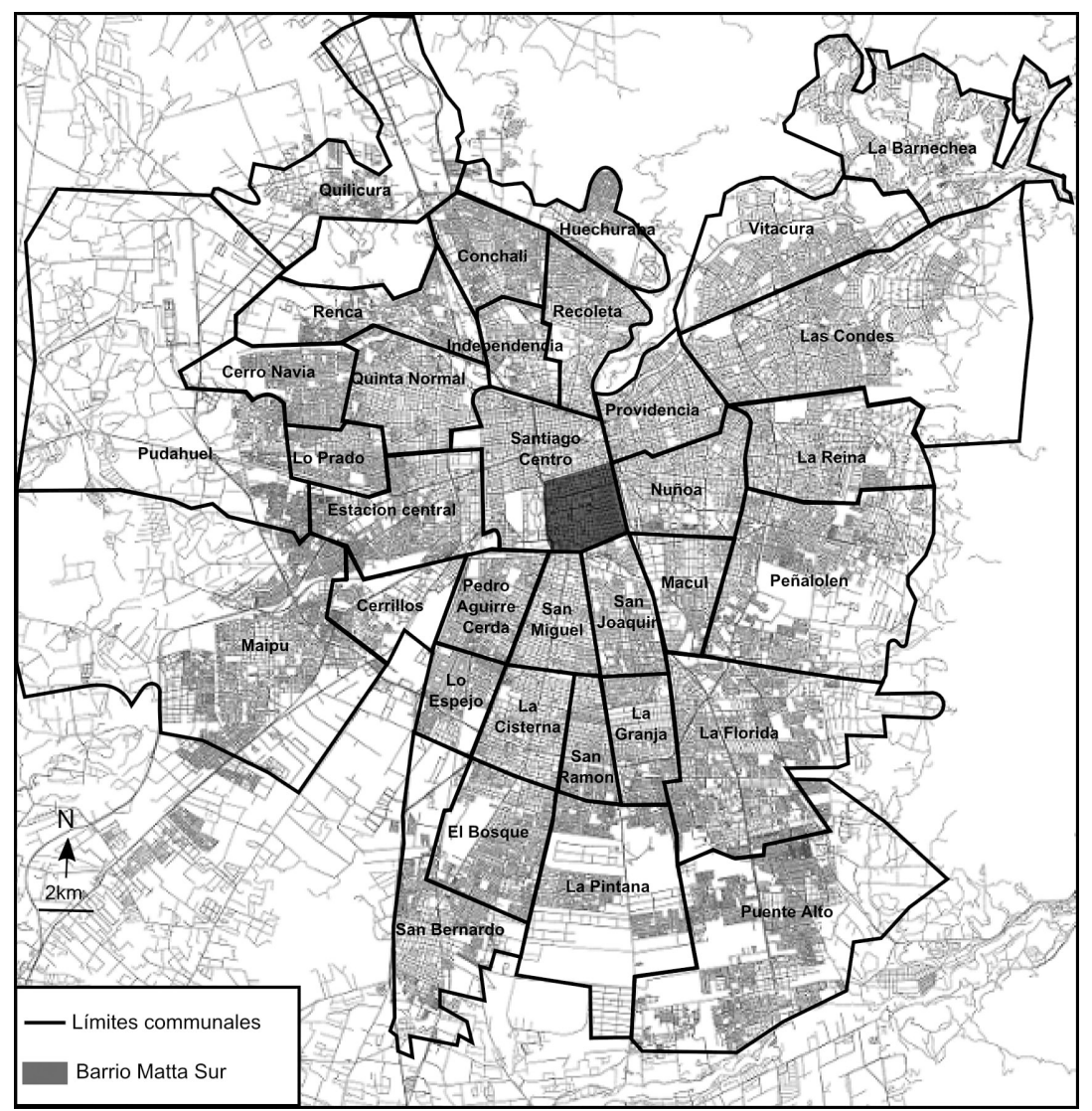

Fuente: Realización propia, 2015. 
El "Comité de Defensa de Matta Sur" fue creado para oponerse al Ministerio de Transporte, que quería construir un corredor de buses en avenida Matta, en el norte del barrio. La movilización fue un éxito pues el proyecto no vio la luz. A partir de este logro, los habitantes han extendido progresivamente sus reivindicaciones a la escala del barrio, con el objetivo de protegerlo frente a la multiplicación de proyectos inmobiliarios en la comuna de Santiago Centro. En este marco, los habitantes del Comité se dieron cuenta que existían otros grupos en el barrio y que compartían sus preocupaciones. Primero se reunieron en un solo grupo llamado "Comité de Defensa y de Recuperación del barrio Matta Sur". Luego se separaron en dos, formando así el "Centro Cultural Patrimonio Matta Sur" en 2009. Mientras el "Comité Matta Sur" se preocupa de la gestión y la protección del barrio, el "Centro Cultural" tiene por misión la animación de actividades en relación con la revalorización patrimonial.

Después de muchos años de movilización, en diciembre de 2015 una parte del barrio fue declarada zona típica ${ }^{6}$ por el Consejo de los Monumentos Nacionales. En este artículo, el análisis se focaliza en el periodo anterior a esta declaración, interesándose en los discursos y las prácticas que condujeron a este logro. En este marco, además de la elaboración de la demanda de protección, los grupos proponían distintas actividades tales como la plantación de árboles y flores en las platabandas de las calles, la organización de eventos culturales en el espacio público o en los centros culturales del barrio, la invitación a charlas sobre el tema patrimonial, la organización de rutas patrimoniales en el barrio y también la elaboración de proyectos más amplios como la creación de un museo del barrio o de un registro de fotografías patrimoniales. Por otro lado, difundían informaciones e ideas mediante un boletín llamado "El Adoquín". Hoy en día, estos grupos siguen trabajando para la revalorización de la "identidad barrial" de Matta Sur y para sensibilizar a sus habitantes y a los residentes de otros barrios acerca de este tema, a través de reuniones informativas, charlas, visitas organizadas en el barrio, eventos culturales y distribución del boletín.

El trabajo se basa en observaciones participantes en reuniones y actividades propuestas por estos grupos. Participamos principalmente en dos proyectos financiados con fondos concursables de la municipalidad o del Estado y coordinados por el "Centro Cultural Patrimonio Matta Sur" y apoyado por el "Comité de Defensa del barrio Matta Sur".

$6 \quad$ La zona típica es una zona de protección patrimonial declarada por el Consejo de los Monumentos Nacionales y que se acompaña de reglamentaciones prescriptivas. 
Asistimos a seis reuniones de dos horas en 2014, en el marco de la creación de un museo itinerante del barrio realizado a partir de la compilación de objetos otorgados por los habitantes y de pancartas con informaciones sobre el barrio, su historia, su arquitectura, sus habitantes, sus lugares típicos y sus personajes. La primera exposición, en febrero de 2015, tuvo lugar en un centro cultural del barrio y la última, en diciembre de 2015, en el hall de la Municipalidad de Santiago. Participamos también en un taller de fotografía patrimonial que se desarrolló entre abril y junio de 2015 (ver figura 2). Asistimos a dos clases de dos o tres horas a la semana realizadas por un profesor profesional para explicar a los treinta habitantes participantes cómo tomar fotografías, con el objetivo de elaborar juntos un registro patrimonial del barrio según distintos temas tales como la arquitectura, las actividades comerciales, artesanales y culturales, los lugares típicos y la cotidianidad de sus habitantes.

En el marco de estas actividades de terreno, conversamos de manera regular con los participantes y organizadores y observamos las situaciones de interacción entre ellos. Estas escenas y estos discursos fueron recogidos para el análisis gracias a la toma de notas durante las reuniones y los encuentros. Las conversaciones informales y las observaciones de terreno son entonces el material sobre lo cual se basan los análisis ulteriores. Para complementar estos datos se realizaron entrevistas en profundidad con los dirigentes de los grupos estudiados. Finalmente, los textos de los documentos difundidos por las agrupaciones de habitantes (boletín, Facebook, Internet) constituyen también una fuente importante para el trabajo de análisis.

A partir de este trabajo de campo se utiliza la nostalgia como categoría analítica para interrogar los mecanismos sociales observados. Para eso se entiende como emoción consciente y socialmente construida, es decir como sentimiento que se enmarca en situaciones correspondientes a marcos convencionales específicos (Kemper, 1978; Hochschild, 1983). Desde este enfoque, la nostalgia permite comprender comportamientos y discursos que se vinculan con ideas y valores inscritos en un tipo de relación subjetiva con el espacio y el tiempo. Por este medio se cuestionarán las dimensiones subjetivas y afectivas del cambio urbano. 


\section{FIGURA 2. ACTIVIDAD EN EL TALLER DE FOTOGRAFÍAS PATRIMONIALES.}

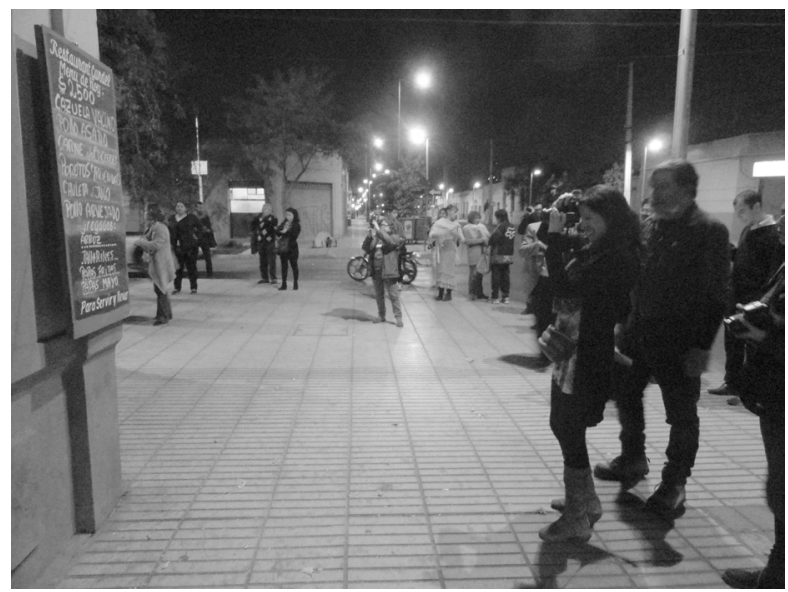

Fuente: Fotografía tomada por el autor, mayo de 2015.

\section{Las condiciones socio- espaciales de la nostalgia en Santiago de Chile}

En la literatura, los autores asocian muchas veces la nostalgia con la idea de aceleración y de pérdida de control sobre los eventos. Starobinski (1966) nota que la transformación en la idea de nostalgia, entendida como deseo de regreso a un tiempo pasado, se enmarca en un contexto de proceso de urbanización que empezó en el siglo XIX. Por su lado, Davis (1979) observa que una cultura de la nostalgia nació en los años 1960-1970 en los Estados Unidos, en una época de cambios sociales y de difusión masiva de estos por los medios de comunicación. Finalmente, otros autores como Appadurai (1996) o Boym (2001) muestran cómo el proceso de globalización y la conformación de un mundo de incertidumbres han generado una epidemia global de nostalgia y han reforzado la afectividad que los habitantes podrían tener por lo local, provocando y activando procesos de defensa identitaria a esta escala.

En Santiago Centro, los procesos de defensa barrial se enmarcan en un periodo de intensificación de los proyectos inmobiliarios y de densificación urbana acelerada que empezó en los noventa. Durante esta década, el Estado decidió detener la extensión urbana, lo que fue una consecuencia de la política liberal de Pinochet de los años ochenta (Vicuña, 2013). Para eso desarrolló un nuevo tipo de urbanismo que podríamos llamar "pro-empresarial" (Harvey, 1989). Es un urbanismo impulsado y gestionado por el Estado y las municipalidades para atraer una parte del sector privado nacional e internacional que busca invertir su capital en el mercado inmobiliario o del suelo (Mattos, 2002; Hidalgo, 2010). En este marco, la Corporación para el Desarrollo de Santiago (Cordesan) institución mixta pública-privada dependiente de la Municipalidad de Santiago- fue creada después del terremoto de 1985 para elaborar planes de repoblamiento en la comuna. Iniciados en 1992, 
tales planes consisten en la delimitación de zonas de renovación con la constitución de reserva de terrenos, la creación de subsidios a la vivienda y la desregulación de las normas de construcción establecidas por el plan regulador de 1939. Para atraer inversionistas y familias en el centro, la municipalidad buscó también mejorar la calidad del espacio público y de las zonas peatonales. En suma, desde los años noventa hasta hoy, la renovación urbana de Santiago Centro se basa en la inversión especulativa del capital privado, en lo cual las municipalidades y el Estado tienen un rol de "facilitador" de mercado.

La renovación urbana aplicada en el centro de Santiago puede ser considerada como una ruptura en la producción de la ciudad. Una de las consecuencias más visible es la verticalización de Santiago Centro, debido a la cada vez más fuerte búsqueda de rentabilización de la producción de vivienda (ver figura 3). Desde los inicios de los planes de repoblamiento se han construido más de 100.000 viviendas en más de 500 operaciones inmobiliarias (Contreras, 2011), destruyendo sectores enteros del centro, reemplazando casas o edificios antiguos con valor patrimonial e histórico por torres que superan a veces treinta pisos (Hidalgo, 2010). Además, los efectos son también sociales porque estos procesos conducen a una renovación de la población. La mayoría de los nuevos residentes son familias monoparentales, solteros o parejas sin hijos que eligen vivir en el centro por su localización cerca del trabajo, por los transportes y por la proximidad de sus redes sociales, profesionales y familiares (Contreras, 2011). Son individuos que construyen sus espacios de socialización afuera del barrio y que no buscan construir relaciones con su entorno cercano. Por lo tanto, son personas que se distinguen de los habitantes de origen en sus costumbres y prácticas socio-espaciales cotidianas, pero también en su concepción del barrio.

Frente a estas transformaciones socio-territoriales, se observa desde los años 2000 la movilización de agrupaciones de vecinos para proteger su espacio de vida. Bajo nombres tales como "Comité de defensa del barrio" o "Vecinos defienden el barrio", estos grupos logran imponer el tema de la defensa barrial en la escena política local y nacional (Canteros, 2011). En 2009, una parte del barrio Yungay fue declarada "zona típica". En 2015 ocurrió lo mismo en el barrio Matta Sur. Además, numerosos sectores son protegidos por la municipalidad como zona de conservación histórica. Finalmente, los planes reguladores de Santiago reglamentan con más prescripciones la altura máxima de los nuevos edificios. Estas evoluciones son favorecidas, entre otros motivos, por el contexto político de la época. A escala comunal, de 2012 a 2016, la Municipalidad de Santiago fue dirigida por responsables políticos que apoyaron las iniciativas de las agrupaciones. En tanto, a escala nacional se puede hacer referencia a algunos programas estatales que participan en la protección barrial. Por ejemplo, el 


\section{FIGURA 3. DENSIFICACIÓN URBANA EN SANTIAGO CENTRO.}

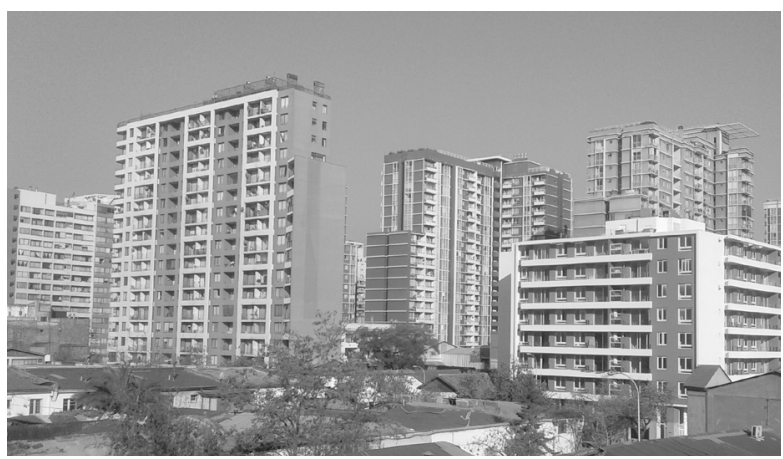

Fuente: Fotografía tomada por el autor, noviembre de 2016.

programa "Quiero Mi Barrio", iniciado en 2006 y conducido por el Ministerio de Vivienda y Urbanismo, consiste en seleccionar algunos barrios en el conjunto de ciudades chilenas con el objetivo de mejorar la accesibilidad y la calidad de vida. En paralelo, grupos de habitantes postulan también a fondos concursables del Consejo Nacional de la Cultura y las Artes, donde la revalorización patrimonial es una temática cada vez más presente.

En paralelo de estas evoluciones, a partir de 2009 la "Asociación Chilena de Barrios y Zonas Patrimoniales" organiza reuniones y conferencias sobre el tema de la protección patrimonial en Chile. Intenta además coordinar las acciones de distintos grupos de vecinos en todo Chile. Esta asociación espera así proponer una nueva ley para crear un Ministerio de Cultura donde el patrimonio tendría un lugar de primera importancia.
A través de sus acciones, los habitantes movilizados quieren entrar en conflicto con los promotores inmobiliarios y más generalmente con el sistema político-económico neoliberal chileno que conduciría, según ellos, a una pérdida de los valores y de los estilos de vida que defienden y que asocian directamente con la idea de barrio.

\section{Del miedo por la pérdida a las prácticas de rememoración}

Los grupos movilizados se estructuran a partir de sus relaciones afectivas mutuas que sienten y expresan por y en el barrio. En el caso de Matta Sur, son relaciones de familias presentes en el barrio a veces desde muchas generaciones. Gran parte de los habitantes han crecido juntos y por lo tanto se conocen. Además, se identifican con el grupo porque se compone de personas que experimentan el mismo tipo de territorialidad. El barrio hace parte de los referentes, de los espacios fundadores (Ramos, 2006) de su identidad individual y colectiva. Progresivamente, durante las reuniones y las entrevistas informales con los participantes se puede comprender que están particularmente interesados y preocupados por la preservación de sus recuerdos y de sus memorias. Para ellos, proteger las dimensiones materiales del barrio significa 
preservar sus memorias, ya que el espacio les sirve para recordarse (Halbwachs, 1950; Bastide, 1970). La construcción de la identidad colectiva del grupo se funda así en un repertorio cultural común entre los participantes, basado en historias y recuerdos compartidos respecto a los lugares de "antes" y donde los discursos están impregnados de nostalgia. Así, el antiguo cine transformado hoy día en local de reparación de autos o el restaurante San Remo destruido en el marco de la instalación de la futura línea de metro son, por ejemplo, temas que aparecen regularmente en las conversaciones. A veces recuerdan y conversan de su cotidiano durante la dictadura y las personas mayores en ocasiones evocan los trolleys que pasaban por las calles del barrio y animaban los lugares o las calles de la época. Cada recuerdo está asociado a un lugar material existente.

En este marco, la inquietud y el miedo a ver su barrio desaparecer o cambiar y sus relaciones vecinales y familiares reducidas a causa de las intervenciones de los promotores inmobiliarios y por la llegada de nuevas familias con costumbres distintas, conducen a los habitantes a expresar públicamente su malestar en y por medio de grupos de defensa barrial. En sus conversaciones comparten un mismo referente: el barrio y, con él, la nostalgia que cada participante siente y valoriza.
Progresivamente, a través de sus prácticas y discursos compartidos, la nostalgia, que era la base de la motivación individual para participar en los grupos, se vuelve colectiva y se comparte entre cada participante. Las interacciones entre miembros del grupo tienen un efecto amplificador de las emociones sentidas individualmente (Livet, 2002). En este marco, la necesidad de transmitir se convierte en una prioridad. En el caso de Matta Sur, la voluntad de transmisión es reforzada por el miedo de perder, no solamente las memorias y recuerdos del barrio, sino también lo que constituye su identidad y su cultura. Los grupos de vecinos añoran lo que falta en el tiempo presente y que es actualmente inaccesible a causa de "la irreversibilidad del tiempo" (Pickering y Keightley, 2006, p. 920)7. Eso se revela en los discursos escuchados, en los comportamientos observados durante la elaboración del museo del barrio y en los talleres de fotografías patrimoniales. Respecto a la primera actividad, el tema de la pérdida o de la desaparición estaba omnipresente. Por eso, la idea de hacer un museo correspondía a la voluntad de rescatar objetos físicos, memorias orales e informaciones, mostrando y explicando el estilo de vida de Matta Sur. En este marco, los habitantes involucrados reconstituyen y reconstruyen una relación con un pasado a partir de las herencias memoriales de sus familias y de sus propios recuerdos para poder otorgarlos a las

7 Traducción del autor. 
próximas generaciones. El taller de fotografías patrimoniales sigue la misma estrategia. No se trata solamente de inmortalizar a través de la fotografía un paisaje urbano en riesgo, sino también rescatar un ambiente urbano y las actividades económicas, sociales y culturales que lo configuran. Anticipan así sus temores de desaparición. La nostalgia les sirve para proyectarse en el presente y el futuro.

En suma, en Matta Sur, la revalorización patrimonial no se vincula con una reinterpretación de la memoria histórica, sino más bien con una reactualización de momentos pasados vividos y anclados en el barrio. Se trata así de un proceso nacido de reminiscencias individuales gatilladas por el cambio urbano que conduce progresivamente a un trabajo de rememoración realizado a partir de los recuerdos y memorias de los habitantes, donde la nostalgia es al mismo tiempo la base y el medio usado por el grupo para rescatar el patrimonio barrial.

\section{La nostalgia como modo de apropiación del barrio}

Los grupos estudiados revalorizan los lugares que consideran como patrimoniales a través de demandas de protección jurídica del barrio, de discursos pronunciados durante las actividades y las reuniones, de la exposición de objetos cotidianos y de fotografías en el museo del barrio. Esta recalificación patrimonial se basa en la nostalgia colectiva sentida y compartida por los miembros del grupo respecto a su espacio de vida. Este sentimiento se traduce en prácticas sociales que pueden ser comparadas a una apropiación simbólica del espacio que, según Veschambre (2008), "supone la producción, el uso de símbolos, dotados de una eficiencia social y política para significar que tal espacio, tal objeto es asociado con un grupo, una institución, un poder" (p. 7) ${ }^{8}$. En el caso de Matta Sur, se observa una apropiación material del espacio, por ejemplo a través de la plantación de flores en las platabandas del barrio. La actividad tiene por objetivo no solamente embellecer las calles, sino también afirmar que este barrio se puede auto-gestionar por sus propios habitantes.

Pero, la apropiación es también ideal, ya que se hace a través de los discursos, de las formulaciones y de las palabras elegidas para caracterizar el barrio. Durante los talleres de fotografías patrimoniales, los habitantes hablan de "nuestro" barrio, de "nuestras" casas, de "nuestras" calles. Los adjetivos posesivos son omnipresentes. En el mismo sentido, durante las entrevistas con los responsables de los grupos de defensa de Matta Sur, ellos explican que su objetivo es la "recuperación" del barrio por sus habitantes. Esta palabra es usada en la mayoría de los proyectos y está presente en el

8 Traducción del autor. 
nombre del comité en sus inicios en 2007: "Comité de Defensa y de Recuperación del barrio Matta Sur". La apropiación se hace también por medio de prácticas cotidianas de vigilancia del espacio público. Cerca de las 18 horas, los habitantes salen a las calles, delante de la puerta de sus casas o de sus vecinos, para conversar. Incluso pasean por las calles en búsqueda de personas con quienes conversar. Los habitantes ocupan el espacio público de las calles y de las plazas en su cotidianidad, lo hacen suyo, por lo que una persona ajena al barrio se sentiría indeseable en este espacio social. Otra manera de vigilar el barrio es por Internet. En la página de Facebook de los grupos es frecuente que algunos habitantes denuncien la construcción de nuevos edificios y señalen las nuevas actividades económicas.

La nostalgia es utilizada como herramienta de apropiación simbólica del espacio en los dispositivos de sensibilización del grupo, que se pueden definir como "el conjunto de soportes, de disposiciones de objetos, de puestas en escenas que los actores desplieguen a fin de suscitar reacciones afectivas que predisponen a aquellos que las experimentan a apoyar la causa defendida" (Traïni, 2015, p. 19) ${ }^{9}$. Además de los proyectos en los cuales participamos, se pueden referir las rutas patrimoniales organizadas por el "Centro Cultural". Consisten en un recorrido por el barrio que dura entre una y dos horas en fines de semana y donde uno de los miembros del centro cuenta las historias de los lugares encontrados en el camino. En una salida en la cual participamos, el animador explicó cómo era el barrio antes, presentándolo como mejor que en el presente. Insistió también sobre la relevancia de proteger los edificios para no solamente conservar los valores históricos, sino además para recordar un tiempo pasado. En su discurso, el patrimonio era claramente relacionado con los habitantes del barrio y sus estilos de vida. Asociando el barrio, su historia y sus memorias con los habitantes, los grupos quieren así mostrar y reivindicar su pertenencia a este territorio, pero también a un tipo de relación nostálgica al tiempo compartida por los participantes y que sirve de base a su construcción identitaria común.

Esta forma de apropiación nostálgica del espacio tiene otra función social: diferenciarse de los nuevos residentes que no comparten los mismos recuerdos, valores y tampoco la misma concepción del barrio. Podríamos caracterizar a estas personas de "móviles", pues no tienen mucho vínculo social, cultural o económico con el barrio. Los habitantes de los grupos de defensa barrial expresan menosprecio por estos nuevos residentes que quieren vivir en edificios con "vista hacia la Cordillera", sin buscar crear relaciones con los "vecinos". Desde las observaciones de terreno, se puede entender

Traducción del autor. 
que los habitantes establecidos buscan valorizar un estilo de vida que tiene que ver con el anclaje identitario local, que se basa en una interpretación de lo que debería ser un barrio a partir de recuerdos o imaginarios respecto a un tiempo pasado. Para confrontarse al estilo "móvil" de habitar de los nuevos residentes, los habitantes establecidos defienden su idea del barrio como un espacio social donde se encuentran habitantes compartiendo los mismos estilos de vida y las mismas formas de sociabilidad. En este sentido, revalorizan el anclaje y el arraigo. Se trata de una revalorización de la "autoctonía" que se hace a través de prácticas socio-espaciales cotidianas, tales como ir a comprar el pan amasado a la misma hora que los vecinos, conversar en la calle con ellos cada día a las 18 horas, reunirse los sábados y domingos en la tarde en la plaza del barrio, o hacer las compras en los comercios locales o en la feria el sábado en la mañana. El mantenimiento de las sociabilidades vecinales parece así ser un elemento central, sin el cual la vida barrial sería imposible.

Por lo tanto, la nostalgia sirve de base emocional común y de herramienta para fomentar la "autoctonía" como estilo de vivir en continuidad con un tiempo pasado. Por este medio, la nostalgia sirve también para reinventar un "orden social" que se basa en ideas y reinterpretaciones de un pasado, para pensarse y proyectarse hacia un futuro donde el anclaje local tiene gran importancia. Quieren dar un sentido al hecho de vivir en el barrio, un sentido no entendido y no compartido por los residentes de las torres y que ayuda a los habitantes establecidos a sentirse pertenecientes al grupo y al barrio.

\section{Conclusión}

A partir de los resultados presentados en este artículo, se puede concluir que la nostalgia produce un espacio particular que podríamos llamar revenant -fantasma- (Certeau, 1980) y que se forma a través de un doble proceso.

El primero es socio-cultural. Se basa en las emociones generadas por la reminiscencia que experimentan los habitantes frente al cambio socioterritorial. En este marco, la nostalgia se materializa de manera efímera a través de apropiaciones puntuales pero cotidianas del espacio. Un segundo proceso es institucional, donde se trata de proteger jurídicamente el espacio barrial por medio de una territorialización del valor patrimonial realizada por los habitantes y las instituciones. Se vincula con la rememoración de un pasado efectuada a partir de una construcción colectiva que se materializa de manera permanente y marca el espacio, no solamente por medio de las reglamentaciones y de las normas de construcción impuestas por las 
instituciones una vez la delimitación de la protección elegida, sino también por las intervenciones de revalorización material que hacen los habitantes en sus casas y en la calle.

Otra conclusión es que la territorialización de la nostalgia se basa en una apropiación material e ideal del barrio que es integradora para los participantes pero excluyente para las personas que no comparten la nostalgia por un tiempo pasado o que no están de acuerdo con esta concepción nostálgica del barrio. La revalorización de un "como era antes" se vincula así con una búsqueda identitaria que conduce al fomento de normas sociales y reglas comportamentales por parte de los habitantes y la estigmatización del otro que no tiene la misma afectividad respecto a las historias y memorias del barrio.

\section{Referencias bibliográficas}

Angé, 0., y Berliner, D. (Eds.). (2015). Anthropology and nostalgia. Oxford, Reino Unido: Berghahn.

Appadurai, A. (1996). Modernity at large. Cultural dimensions of globalizations. Minneapolis, Estados Unidos: University of Minnesota Press.

Bastide, R. (1970). Mémoire collective et sociologie du bricolage. L’Année sociologique, (21), 65-108.
Bauman, Z. (2007). Liquid time: Living in an age of incertainly. Cambridge, Reino Unido: Polity Press.

Berdahl, D. (2010). (N)ostalgie for the present. Memory, longing, and East German things. Ethnos, 64(2), 192-211. https://doi.org/10.1080/00141844.1999. 9981598.

Berliner, D. (2012). Multiple Nostalgias: The fabric of heritage in Luang Prabang. The Journal of the Royal Anthropological Institute, 18(4), 769-86. https://doi. org/10.1111/j.1467-9655.2012.01791.x.

Bissell, W. (2005). Engaging colonial nostalgia. Cultural Anthropology, 20(2), 215-248. https://doi. org/10.1525/can.2005.20.2.215.

Boym, S. (2001). The Future of nostalgia. New York, Estados Unidos: Basic Books.

Canteros, E. (2011). Las agrupaciones vecinales en defensa de los barrios. La construcción política desde lo local. Polis, 10(28), 85-99. https://doi. org/10.4067/S0718-65682011000100006.

Certeau, M. d. (1980). L'invention du quotidien (Tome 2). Paris, Francia: Gallimard.

Contreras, Y. (2011). La recuperación urbana y residencial del centro de Santiago: Nuevos habitantes, cambios socioespaciales significativos. EURE, 37(112), 89-113. https://doi.org/10.4067/ S0250-71612011000300005.

Davidson, J., Bondi, L., y Smith, M. (Eds.). (2007). Emotional geographies. Aldershot, US: Ashgate.

Davis, F. (1979). Yearning for yesterday. A sociology of nostalgia. New York, Estados Unidos: Free Press. 
Elias, N., y Scotson, J. (1966). The Established and the Outsiders: A sociological enquiry into community problems. London, Reino Unido: Frank Cass \& Co. Ltd.

Gervais-Lambony, P. (2012). Nostalgies citadines en Afrique du Sud. EspacesTemps.net. Recuperado de http://www.espacestemps.net/articles/ nostalgies-citadines-en-afrique-sud/.

Halbwachs, M. (1950). La mémoire collective. Paris, Francia: Presses Universitaires de France.

Harvey, D. (1989). From managerialism to entrepreneurialism: the transformation in urban governance in late capitalism. Geografiska Annaler, 20(3), 233-249.

Harvey, D. (1990). The condition of Postmodernity. Oxford, Reino Unido: Blackwell.

Hemer, S. R., y Dundon, A. (Eds.). (2016). Emotions, senses, spaces: ethnographic engagements and intersections. Adelaide: University of Adelaide Press.

Hidalgo, R. (2010). Los centros históricos y el desarrolo urbano inmobiliario: las contradicciones de un negocio exitoso en Santiago de Chile. Scripta Nova, 14(331), 85. Recuperado de http://www. ub.edu/geocrit/sn/sn-331/sn-331-85.htm.

Hidalgo, R., y Janoschka, M. (Eds.). (2014). La ciudad neoliberal: gentrificación y exclusión en Santiago de Chile, Buenos Aires, Ciudad de México y Madrid. Santiago, Chile: Pontificia Universidad Católica de Chile.
Hochschild, A. (1983). The management heart: Commercialization of human feeling. 3a ed. Oakland, Estados Unidos: University of California Press.

Huyssen, A. (2003). Present pasts, urban palimpsest and the politics of memory. Stanford, Estados Unidos: Stanford University Press. 192 p.

Ilustre Municipalidad de Santiago. (2013). Memoria explicativa: proyecto de modificación al plan regulador de Santiago, sector $n^{\circ} 5$ Matta-Franklin y actualización del texto de la ordenanza local. Santiago, Chile: Autor. 102 p.

Kemper, T. (1978). A social interactional theory of emotions. Hoboken, Estados Unidos: John Wiley \& Sons Inc.

Lachenal, G. y Mbodj-Pouye, A. (2014). Restes du développement et traces de la modernité en Afrique. Politique Africaine, 3(135), 5-21.

Lefebvre, H. (1974). La production de l'espace. Paris, Anthropos. 485 p.

Livet, P. (2002). Émotions et rationalité morale. Paris, Presses Universitaires de France.

López Morales, E., Gasic Klett, I., y Meza Corvalán, D. (2012). Urbanismo pro-empresarial en Chile: políticas y planificación de la producción residencial en altura en el pericentro del Gran Santiago. Revista INVI, 27(76), 75-114. https://doi.org/10.4067/ S0718-83582012000300003.

Lowenthal, D. (1998). The heritage crusade and spoils of history. Cambridge: Cambridge University Press. 338 p. 
Mattos, C. d. (2002). Santiago de Chile de cara a la globalización: iotra ciudad? Revista de sociología política, (19), 31-54. https://doi.org/10.1590/ S0104-44782002000200004.

(2010). Globalización y metamorfosis metropolitana en América Latina: de la ciudad a lo urbano generalizado. Revista de Geografía Norte Grande, (47), 81-104. https://doi.org/10.4067/ S0718-34022010000300005.

Pickering, M., y Keightley, E. (2006). The modalities of nostalgia. Current Sociology, 54(46), 919-941. https://doi.org/10.1177/0011392106068458.

Ramón, A. d. (2000). Santiago de Chile (1541-1991): Historia de una sociedad urbana. Santiago, Chile: Sudamericana.

Ramos, E. (2006). L'invention des origines, Sociologie de l'ancrage identitaire. Paris, Francia: Armand Colin.

Rodaway, P. (1994). Sensuous geographies: body, sense and place. London, Reino Unido: Rootledge.

Rodríguez, A., y Rodríguez, P. (2009). Santiago, una ciudad neoliberal. Quito, Ecuador: Organización Latinoamericana y del Caribe de Centros Históricos.

Ricoeur, P. (2000). La mémoire, l'histoire, l’oubli. Paris, Francia: Seuil.

Sennett, R. (1998). The corrosion of character: The personal consequences of work in the new capitalism. New York, Estados Unidos: W.W. Norton \& Company.

Smith, M., Davidson, J., Cameron, L., y Bondi, L. (Eds.). (2009). Emotion, place and culture. Surrey, Reino Unido: Ashgate.
Starobinski, J. (1966). Le Concept de Nostalgie. Diogène, (54), 92-115.

Stewart, K. (1988). Nostalgia - a Polemic. Cultural Anthropology, 3(3), 227-41. https://doi.org/10.1525/ can.1988.3.3.02a00010.

Todorov, T. (2004). Les abus de la mémoire. Paris, Francia: Arléa.

Traïni, C. (Dir.). (2015). Emotions et expertises: les modes de coordination des actions collectives. Rennes, Francia: Presses Universitaires de Rennes.

Tuan, Y-F. (1977). Space and Place. The perspective of experience. Minneapolis, Estados Unidos: University of Minnesota Press.

Veschambre, V. (2008). Traces et mémoires urbaines, enjeux sociaux de la patrimonialisation et de la démolition. Rennes, Francia: Presses Universitaires de Rennes.

Vicuña del Río, M. (2013). El marco regulatorio en el contexto de la gestión empresarialista y la mercantilización del desarrollo urbano del Gran Santiago, Chile. Revista INVI, 28(78), 181-220. https:// doi.org/10.4067/S0718-83582013000200006. 